\title{
The Cotton miR477-CBP60A Module Participates in Plant Defense Against Verticillium dahlia
}

\author{
Guang Hu, ${ }^{1,2,3}$ Mengyan Hao, ${ }^{1}$ Le Wang, ${ }^{1}$ Jianfen Liu, ${ }^{1}$ Zhennan Zhang, ${ }^{1}$ Ye Tang, ${ }^{1}$ Qingzhong Peng, ${ }^{3}$ \\ Zuoren Yang, ${ }^{2, \dagger}$ and Jiahe $\mathrm{Wu}^{1,2,3, \dagger}$ \\ ${ }^{1}$ The State Key Laboratory of Plant Genomics, Institute of Microbiology, Chinese Academy of Sciences, Beijing 100101, China \\ ${ }^{2}$ Zhengzhou Research Base, State Key Laboratory of Cotton Biology, Zhengzhou University, Zhengzhou 450001, China \\ ${ }^{3}$ College of Biology and Environmental Sciences, Jishou University, Jishou, Hunan 416000, China
}

Accepted 22 December 2019.

\begin{abstract}
Previous reports have shown that, when Verticillium dahliae localizes at the root surface, many microRNAs (miRNAs) were identified at the early induction stage. Here, we constructed two groups from two timepoints of small RNA (SRNA) in cotton root responses to $V$. dahliae at the later induction stage, pathogen localizing in the interior of root tissue. We identified 71 known and 378 novel miRNAs from six libraries of the pathogen-induced and the control sRNAs. Combined with degradome and sRNA sequencing, 178 corresponding miRNA target genes were identified, in which 40 target genes from differentially expressed miRNAs were primarily associated with oxidation-reduction and stress responses. More importantly, we characterized the cotton miR477CBP60A module in the later response of the plant to $V$. dahliae infection. A $\beta$-glucuronidase fusion reporter and cleavage site analysis showed that ghr-miR477 directly cleaved the messenger RNA of GhCBP60A in the posttranscriptional process. The ghrmiR477-silencing decreased plant resistance to this fungus, while the knockdown of $G$ CBP60A increased plant resistance, which regulated GhICS1 expression to determine salicylic acid level. Our data documented that numerous later-inducible miRNAs in the
\end{abstract}

Guang $\mathrm{Hu}$, Mengyan Hao, and Le Wang are co-first authors.

Accession numbers are as follows: AtCBP60a to AtCBP60g (AT5G62570, AT5G57580, AT2G18750, AT4G25800, AT2G24300, AT4G31000 and AT5G26920), AtSARD1 (AT1G73805), GhCBP60A (XM_016872607), GhICS1 (XM_016873229), GhACT4 (AY305726), GhUBQ7 (DQ116441), VdTUB1 (KF555285), NbUBI (Niben101Scf01953g01013.1), and GhPRI (XM_016825572). The raw data involved in this research has been uploaded to the Sequence Read Archive of the GenBank database with accession numbers SRR10572980, SRR10572981, and SRR10572982.

The ghr-miR477 participates in cotton plant defense.

${ }^{\dagger}$ Corresponding authors: J. Wu; wujiahe@im.ac.cn and Z. Yang; yangzuoren4012@163.com

Funding: This work was supported by the National Transgenic Major Project of China (2018ZX0800901B and 2016ZX08005-003-002) and sponsored by the National Natural Science Foundation of China (31771848 and 31971905).

*The $\boldsymbol{e}$-Xtra logo stands for "electronic extra" and indicates that supplementary figures and supplementary tables are published online.

The author(s) declare no conflict of interest.

(c) 2020 The American Phytopathological Society plant response to $V$. dahliae, suggesting that these miRNAs play important roles in plant resistance to vascular disease.

Keywords: calmodulin-binding protein, Gossypium hirsutum L., later response, miR477, Verticillium dahliae Kleb, vascular disease

Cotton (Gossypium hirsutum L.) is a vital textile and oil crop in the world, but its productivity is constrained by various biotic and abiotic stresses (Xie et al. 2015). One of the stresses is verticillium wilt, a highly destructive vascular disease primarily caused by the soil-borne fungus Verticillium dahliae Kleb (Bhat and Subbarao 1999). The representative symptoms of diseased cotton plants include leaf curl, necrosis and defoliation, and stem wilt (Sink and Grey 1999). V. dahliae generally enters the vascular tissue through wounded root sites and colonizes and grows in xylem vessels (Bejarano-Alcázar et al. 1997; Klosterman et al. 2009). Although no disease symptoms are evident at the stage of pathogen colonization in the xylem vessels, physiological and biochemical status should result in remarkable changes in the root cells, especially those around the vascular tissues, resulting from a substantial amount of gene expression reprogramming at the transcription and translation levels.

microRNAs (miRNAs) are important components in the posttranscriptional regulation of target gene expression, playing major roles in plant development and stress responses (JonesRhoades et al. 2006). miRNAs can recognize corresponding messenger RNA (mRNA) targets based on sequence complementarities and guide, individually or together, the direct cleavage of target mRNAs and translational repression (Li et al. 2013). Recently, miRNA-mediated gene silencing was found to play a significant role in plant defense against pathogens (Khraiwesh et al. 2012; Shriram et al. 2016). For example, Arabidopsis miR393 was the first miRNA discovered to be involved in plant immunity (Navarro et al. 2006). Overexpressing both miR160a and miR398b in rice (Oryza sativa) increased plant resistance to Magnaporthe oryzae, as demonstrated by decreased fungal growth and the upregulated expression of defense-related genes in transgenic plants (Li et al. 2014). In cotton plants infected with the fungus, the production of miR166 and miR159 was increased and was output into the fungal hyphae of $V$. dahliae for specific silencing (Zhang et al. 2016). When miR482 was silenced in cotton plants, expression of the NBS-LRR (nucleotide binding site-leucine rich repeat) defense genes was upregulated, resulting in increasing resistance to fungal pathogen attack (Zhu et al. 2013). Recently, the cotton miR5272a-mediated regulation of MKK6 transcription 
contributes to the cotton plant immune response (Wang et al. 2017a). The evidences are demonstrated in the participation of the miRNAs in cotton plant defense, but the molecular mechanisms and mode of regulation of miRNAs and their corresponding target genes are still unclear.

Calmodulin $(\mathrm{CaM})$ is the most prominent $\mathrm{Ca}^{2+}$ transducer in eukaryotic cells, regulating the activity of numerous proteins with diverse cellular functions (Bouché et al. 2005; Yuan et al. 2017). The CaM-binding protein 60 (CBP60) family contains eight members, CBP60a to CBP60g and SYSTEMIC ACQUIRED RESISTANCE DEFICIENT 1 (Wang et al. 2009, 2011). Three members, CBP60a, CBP60g, and SARD1, have roles in immune signaling. CBP60a encodes a negative regulator of immunity (Truman et al. 2013). The cbp60a mutation is more resistant to Pseudomonas syringe pv. maculicola, and infected plants have higher levels of salicylic acid (SA). Several immune-responsive genes including ICS I/SID2 (ISOCHORISMATE SYNTHASE 1, SA INDUCTIONDEFICIENT $2)$, an enzyme required for SA synthesis, is expressed at higher levels than the wild type in mock-infected cbp60a mutants (Truman et al. 2013). CBP60g and SARD1 are partially function redundant, encoding positive regulators of plant immunity that promote the production of SA (Wang et al. 2011). Thus, it is important to further characterize the cotton CBP60a in plant defense.

miRNA expression sequencing in the early response of cotton plants to $V$. dahliae infection has been reported (He et al. 2014; Yin et al. 2012; Zhang et al. 2015a). For example, Yin et al. (2012) investigated the transcriptional profile of the miRNAs in verticillium-inoculated cotton roots at 12 and 24 h. Two small RNA (sRNA) were constructed from seedlings of the upland cotton variety $\mathrm{KV}-1$ inoculated with $V$. dahliae at 24 and $48 \mathrm{~h}$ ( $\mathrm{He}$ et al. 2014). Zhang et al. (2015a) conducted sRNA sequencing and degradome sequencing of cotton roots inoculated with $V$. dahliae at $24 \mathrm{~h}$. However, in the later induction stage of fungal-infected plants, when $V$. dahliae has colonized in xylem vessels, the response of miRNA expression has not been investigated using miRNA sequencing.

In this study, we investigated sRNA expression profiles in cotton roots inoculated by $V$. dahliae at 7 and 10 days postinoculation (dpi) and mock-treated plants. The results showed that 71 known miRNAs and 378 novel miRNAs were identified from six sRNA libraries, and 40 corresponding target genes from differentially expressed miRNAs were found coupling with degradome sequencing. In the later induction stage, many miRNAs showed a significant difference in their expression level compared with the mock control. Among these differentially expressed miRNAs, a novel ghr-miR477 and its target gene $G h C B P 60 A$ were found to form a module to participate in plant resistance to $V$. dahliae. These findings reveal a miRNAmediated regulatory network with a critical role in the plant response of pathogen infestation in the main battlefield of vascular tissues.

\section{RESULTS}

\section{V. dahliae colonization and growth in the root interiors.}

In our previous studies, cotton plants started to show disease symptoms 15 to 18 days after inoculation with $V$. dahliae, including yellow leaves, defoliation, and stunted growth. However, before the presence of the disease symptoms, it is unclear how plants resist colonization and the upward dispersion of fungi in the interior of plants (primarily xylem vessels). To investigate the colonization of the pathogen and its spread in xylem vessels, root samples from seedlings inoculated with $V$. dahliae for $1,4,7,10$, and 13 days were first treated by ultrasound to remove the fungal hyphae and spores on the root surface. According to quantitative PCR (qPCR) analysis, the DNA of $V$. dahliae in ultrasound-treated cotton roots was barely detectable at 1 and $4 \mathrm{dpi}$, while amounts of fungal DNA in ultrasound-untreated roots was found (Fig. 1A; Supplementary Fig. S1). While, at $7 \mathrm{dpi}$, a few fungal DNA were monitored in the ultrasound-treated roots, a value of $1.03 \times$ $10^{-4}$ compared with the cotton DNA copies; at 10 and $13 \mathrm{dpi}$, relative DNA copies of the fungal pathogen were approximately $1.25 \times 10^{-3}$ and $1.52 \times 10^{-3}$, respectively (Fig. 1A). A fungal recovery assay of the $V$. dahliae-infected root fragments was conducted as a parallel experiment. Consistent with the results of the fungal DNA analysis, fungi were not observed around the root sections at 1 and $4 \mathrm{dpi}$, while approximately $5 \%$ of the root fragments showed fungi growth at $7 \mathrm{dpi}$, and a few fragments at 10 and 13 dpi demonstrated recoverable growth of the fungi (Fig. $1 \mathrm{~B}$ and $\mathrm{C}$ ). These results suggested that $V$. dahliae had colonized in the inside of the roots after $7 \mathrm{dpi}$. To evaluate the interaction of the plant with the pathogen associated with miRNA regulation function in the root vascular tissue, the fungal-treated roots at the two timepoints, 7 and 10 dpi, were chosen for sRNA high-throughput sequencing analysis.

\section{High-throughput sequencing of sRNA.}

To characterize the sRNA profiles in cotton plants challenged by $V$. dahliae infection, six sRNA libraries were constructed, using total RNA isolated from root samples treated with $V$. dahliae for 7 and 10 days and the mixed mock treatment (the control $[\mathrm{CK}]$ ) with two biological replicates. The six libraries were sequenced with an Illumina HiSeq 2500, and a schematic flow of the sequencing and data analysis strategy is shown in Supplementary Figure S2. All six laboratories, including the first repeat of $7 \mathrm{dpi}(\mathrm{Vd} 7 \mathrm{~d}-1)$, Vd7d-2, Vd10d-1, Vd10d-2, CK-1, and CK-2, generated 21,290,628, $19,153,356,35,201,913,35,006,365,34,773,764$, and 33,527,468 clean reads, respectively (Fig. 2A; Supplementary Table S1). Through annotation analysis with the Silva, GtRNAdb, Rfam, and Repbase databases, the sRNAs were grouped into several classes: repeat bases, ribosomal RNA (rRNA), transfer RNA (tRNA), small nucleolar RNA (snoRNA), and unannotated sRNA (Supplementary Table S1). Before analyzing the miRNA, the unannotated sRNA was mapped to the $G$. hirsutum cv. TM-1 genome. A total of 3,937,104, 3,614,802, 4,065,407, 3,822,781, $4,763,073$, and 5,016,335 reads in the six libraries of 7- and 10-dpi inoculated and the control treatments, with two repeats, were successfully matched back to the AD genome, and the mapping rates were $18.49,18.87,11.55,10.92,13.7$, and $14.96 \%$, respectively (Supplementary Table S1). The percentages of genome mappings in the positive and negative chains among six samples were similar (Fig. 2B). Although the total reads of the 7-dpi sample were less than those of the 10-dpi sample and the control, the numbers of mapped unannotated reads containing miRNA were similar among the three treatments (Supplementary Table S1).

To investigate the size distribution of all the sequences, the sequences with sizes between 18 and 30 nucleotides (nt) were determined in the number of matched unannotated reads. The size distribution for the matched reads was similar through the observation of the six libraries, in which the 24-nt reads accounted for the majority, followed by 21-nt reads (Fig. 2C). The results of the sRNA abundance and size in cotton were consistent with previous reports in cotton (Wang et al. 2016) and consistent with the results reported in Arabidopsis thaliana (Rajagopalan et al. 2006) and Glycine max (Song et al. 2011), suggesting that the sRNAs in plants are mainly composed of 21 and 24 nt reads. 


\section{Identification of the miRNAs.}

By using miRDeep2 analysis, we screened the unannotated sRNA sequences to identify miRNAs according to the criteria for the selection of a length of at least $18 \mathrm{nt}$ and a maximum of two mismatches compared with all known plant miRNA sequences in the six libraries. After removing the repeat sequences, a total of annotated known miRNAs in Vd7d-1, Vd7d2, Vd10d-1, Vd10d-2, CK-1 and CK-2 were 70, 69, 69, 70, 70, and 68, respectively (Supplementary Table S2). The two biological repeat libraries were merged to obtain the mean value for further analysis. A total of 71 annotated known miRNAs were found in the three treatments, belonging to 46 miRNA families (Supplementary Table S3). Of the 71 miRNAs across the three treatments, 69 miRNAs were commonly present in all three treatments, while ghr-miR7497 and ghr-miR399e were absent in the 7- and 10-dpi treatments, respectively (Fig. 2D, left panel). Each of the 46 miRNA families contained one to four members. Three families, MIR156, MIR2949, and MIR482, possessed four members, while there were 30 other miRNA families with only one member (Supplementary Figure S3). In addition, among the 46 miRNA families, 21- and 24-nt-long miRNAs were the most-represented sizes, reaching 38.03 and $32.39 \%$, respectively, followed by the 20-nt-long miRNAs $(14.08 \%)$ (Supplementary Table S4).

To identify novel miRNAs, the unannotated sRNAs, which could be mapped to the cotton AD genome excluding the known miRNA, were screened using miRDeep2 software. The six libraries of Vd7d-1, Vd7d-2, Vd10d-1, Vd10d-2, CK-1, and CK-2 contained 371, 368, 367, 370, 372, and 370 novel miRNAs, respectively. The data of novel miRNAs in two repeats was merged, using the mean value to carry out analysis hereafter. A total of 378 novel miRNAs with high confidence were found in the three treatments. There were 373,372 , and 377 novel miRNAs in the 7- and 10-dpi treatments and the control, respectively. Among these 378 novel miRNAs, approximately 367 were common across all three treatments, and only one novel specific miRNA (novel miR_A02_1323) was detected in the control. The novel miR_D09_31005 was only found in the two timepoint treated roots (Fig. 2D, right panel).

In this study, nucleotide bias at positions in the total miRNAs was analyzed to understand the miRNA sequence law. The results demonstrated that the first nucleotide of the miRNAs exhibited a preference for uracil (U) shown in Figure 2E (left panel), consistent with the results from many species, possibly due to miRNA sequence conservation. In addition, Figure $2 \mathrm{E}$ (right panel) shows nucleotide bias, at each position, consistent with other plants (Mi et al. 2008).

miRNA expression response to $V$. dahliae infection and the corresponding target prediction.

The identified miRNAs with more than an average of five transcripts per million (TPM) expression levels were chosen for an analysis of differential expression. As shown in Supplementary Table S5, 28 of 71 (39.44\%) known miRNAs and 148 of $378(39.15 \%)$ novel miRNAs were differentially expressed in the two pathogen-induced treatments compared with the mock-treated control (absolute value of $\log _{2}$ ratio $\geq 1$ ). Among all the differentially expressed miRNAs, five and 29 differentially expressed miRNAs, respectively, from the known and novel miRNAs, were found in both the 7- and 10dpi treated roots (Fig. 3A). Twenty-nine miRNAs showed significant upregulation or downregulation of expression at the two timepoints in treated roots (Cluster 1 and 3); four miRNAs exhibited upregulated expression in the 7-dpi treated roots and downregulation in the 10-dpi treated roots, and only one miRNA showed a contrasting trend (Cluster 2). To verify the result, we performed qPCR analysis. The result supported these miRNA sequencing data (Supplementary Fig. S4).
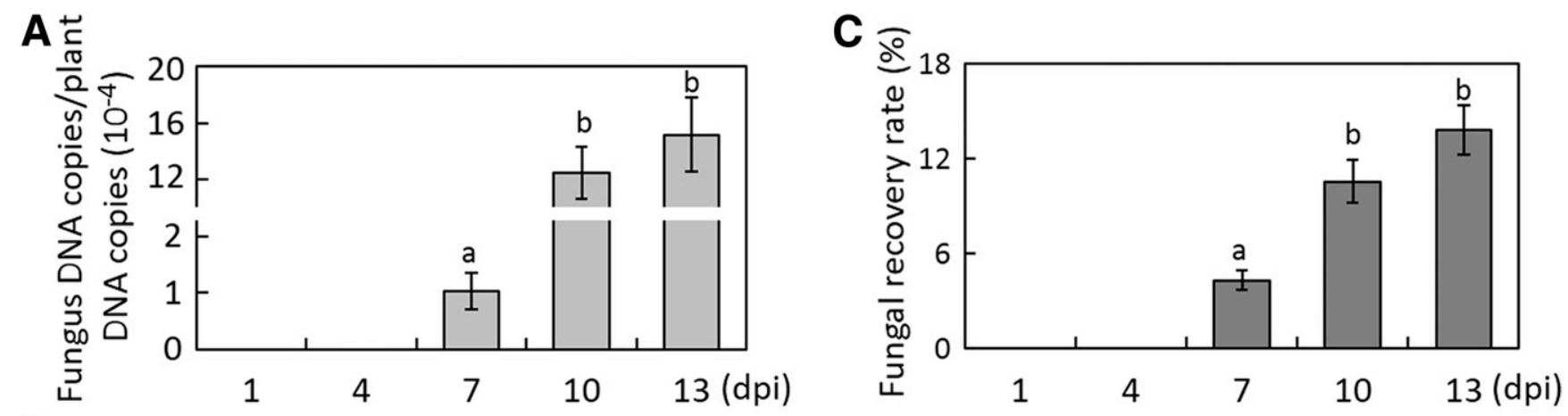

B

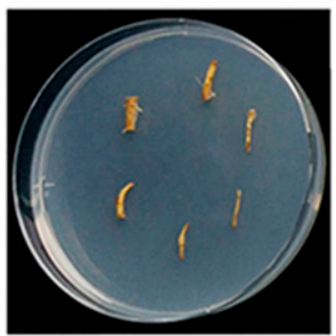

1

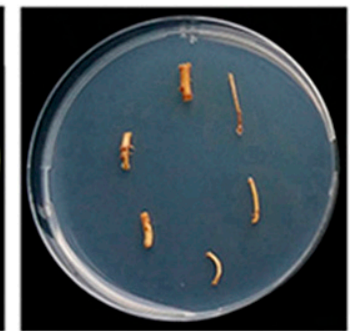

4

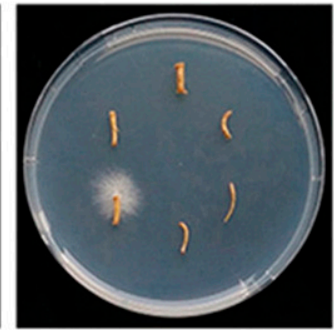

7

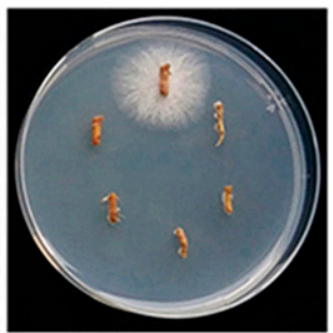

10

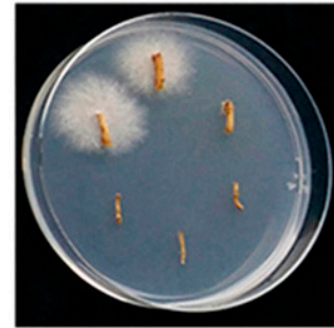

13 (dpi)

Fig. 1. The time course of Verticillium dahliae colonization and growth in the interior of cotton roots. A, Fungal DNA copies versus plant DNA copies in roots inoculated with $V$. dahliae with ultrasound treatment. Quantitative PCR analysis was conducted to compare the DNA contents between the TUB1 gene of V. dahliae and the ACT4 gene of cotton; dpi = day postinoculation. B, Fungal recovery growth from the root fragments placed on potato dextrose agar media at different timepoints of fungal inoculation. Photos were taken 5 days after plating. $\mathbf{C}, V$. dahliae recovery rate of the root fragments $(n=90)$. Error bars in A and $\mathrm{C}$ represent the standard deviation of three biological replicates, different letters indicate significant differences $(P<0.05)$ based on Tukey's honestly significant differences test. 
To further investigate the function of differentially expressed miRNAs, the corresponding target genes were predicted, and gene ontology (GO) enrichment was performed. According to TargetFinder software and the GO classifications, the 405 target genes of the differentially expressed miRNAs were predicted and associated with many GO terms in the 7- and 10-dpi treatments compared with the control, primarily including binding and oxidoreductase activity (Supplementary Tables S6 and S7; Supplementary Fig. S5A and B).

\section{Identification and functional enrichment}

of the miRNA targets by degradome sequencing.

To identify the target genes from a total of 449 miRNAs, degradome sequencing was performed. A total of 178 target genes of the miRNAs were identified. Twenty-five of 71 known miRNAs regulated 75 target transcripts, and 172 of 378 novel miRNAs possessed 142 target genes (Supplementary Tables S8 and S9). miRNAs were able to target various numbers of genes, with a range of one to 16 , of which novel miR D05_23410 targeted the highest number of genes, reaching 16 different genes (Supplementary Tables S8 and S9). As shown in Figure 3B, four novel miRNAs were chosen to analyze their targets combined with degradome sequencing. Their targets, 'target plots' (T-plots), come from the degradome database and describe the cleavage sites of the target sequences by the action of different miRNAs.

Among the 178 target genes, 40 target genes were associated with the differentially expressed miRNAs. According to the GO classifications, the 40 target genes predominantly participated in 61 biological process categories, 53 molecular function categories, and 16 cellular component categories (Supplementary Table S10; Supplementary Fig. S6). Most specific GO classifications showed that the target genes were involved in the oxidation-reduction process, biotic and abiotic stress, and binding (Supplementary Table S10). The Kyoto Encyclopedia of Genes and Genomes (KEGG) analysis
A

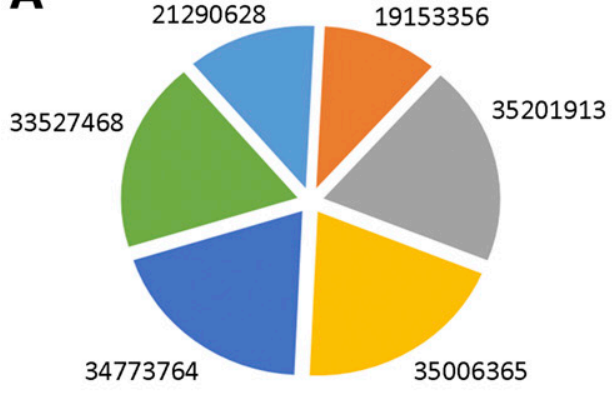

C

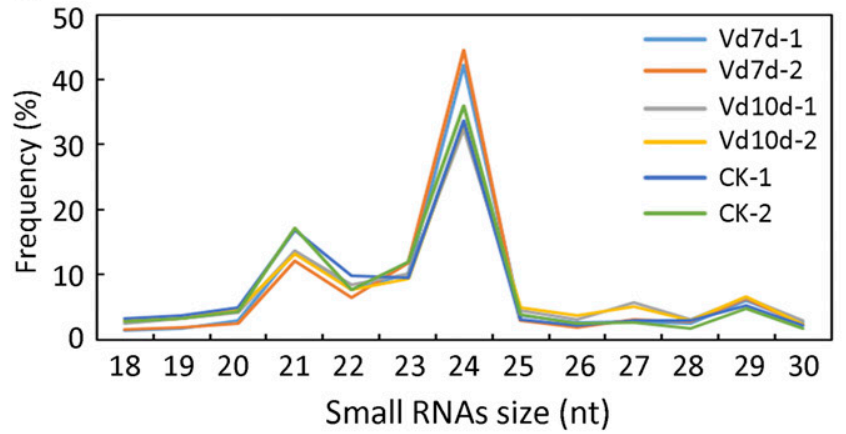

B

Vd7d-1

$V d 7 d-2$

Vd10d-1

Vd10d-2

CK-1

CK-2

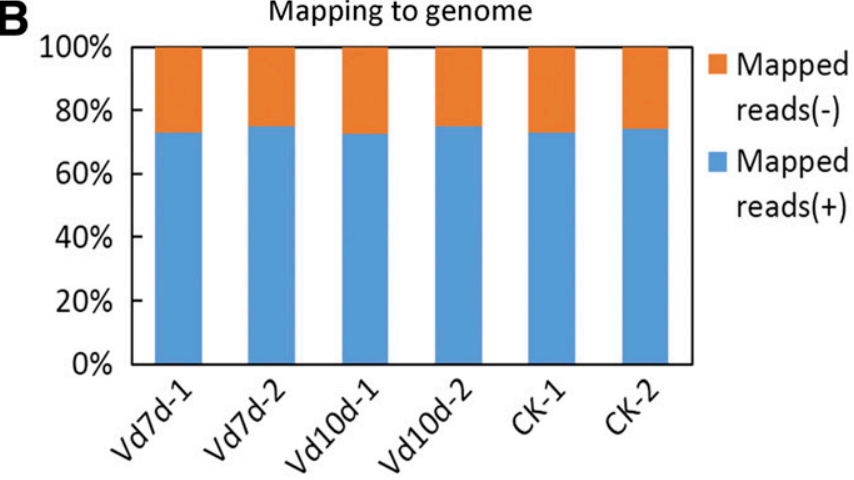

$\mathbf{E}$

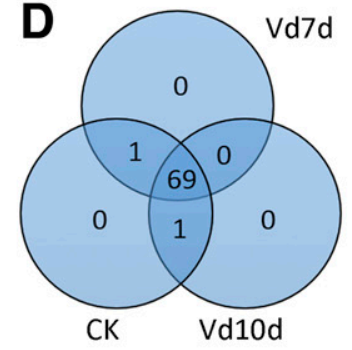

Known miRNA

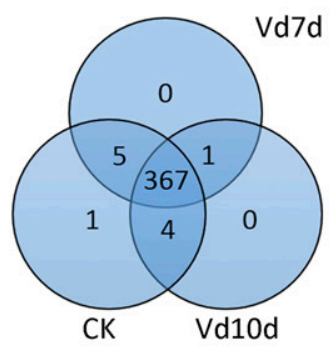

Novel miRNA
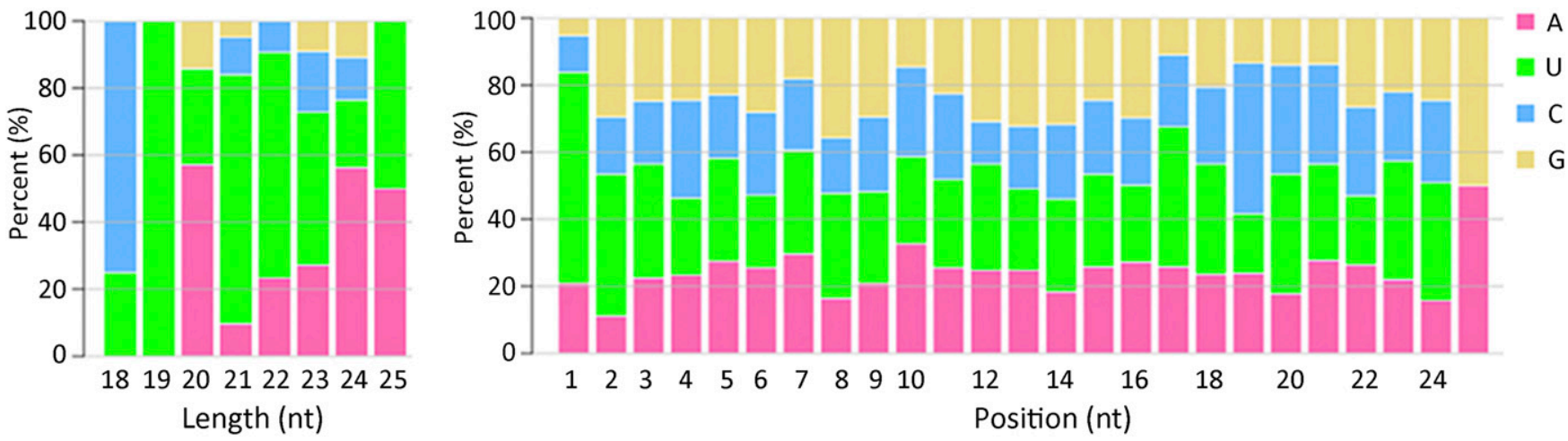

Fig. 2. Small RNA (sRNA) and microRNA (miRNA) analyses of the 7- and 10-days postinoculation (dpi) treatments and the control. A, Total sRNA reads distribution in six samples after removing poor-quality data. B, Total sRNAs mapping to the cotton genome for six samples. Mapped reads(+) and Mapped reads(-) denote the number of clean reads on the cotton genome positive and negative chains, respectively. $\mathbf{C}$, Size distribution of the matched sRNA reads in cotton. D, Venn diagram for special and common known and novel miRNAs among the three treatments. E, miRNA first nucleotide bias (left panel) and miRNA nucleotide bias at each position (right panel) among the three sRNA. Vd7d and Vd10d represent 7 and 10 days after Verticillium dahliae infection; CK, mixed mock treatment samples at 7 and 10 days. 
classified 11 different expression miRNA targets into 10 pathways, and the significantly enriched pathways included terpenoid backbone biosynthesis, carotenoid biosynthesis, and spliceosome (Supplementary Table S11).
Expression authenticity of miRNAs using qPCR.

To further confirm the authenticity of the sRNA highthroughput sequencing, the expression abundance of the miRNAs and their corresponding target genes was tested by

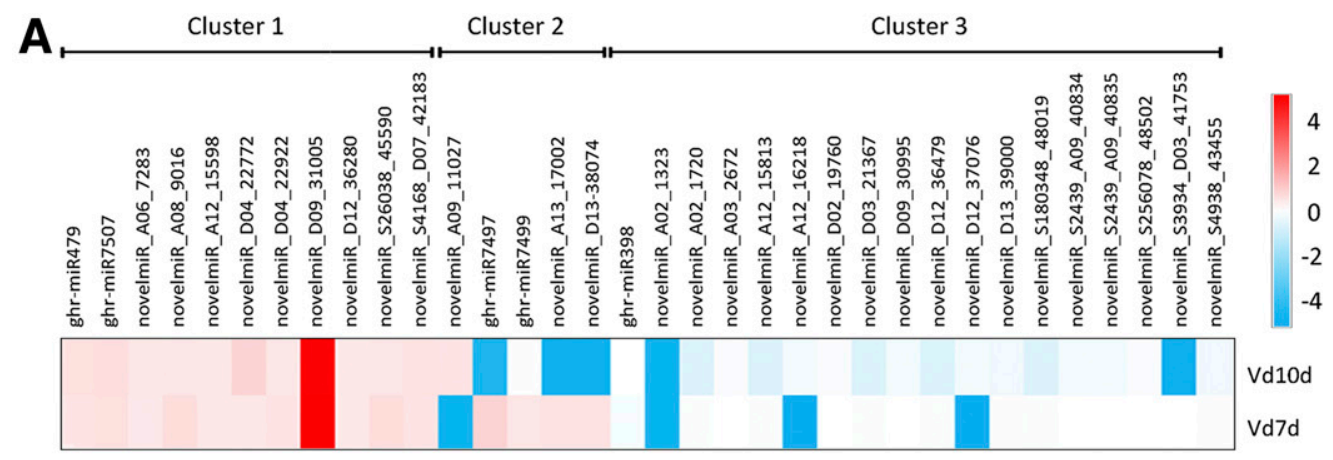

B novelmiR_D04_22772 slicing Gh_A08G0037 at nt 772 Alignment score $=5$, category $=1, p=0.005$

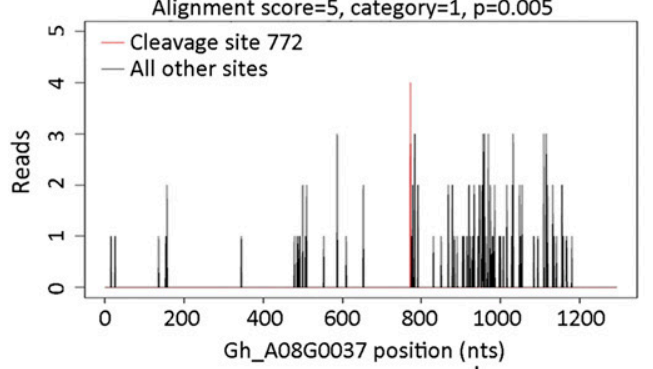

Gh_A08G0037: 5'- CAACGU CACGCGCGAAGCAG -3' novelmiR_D04_22772: $: \begin{aligned} & : \text { '-GCAGCA } \\ & \text { GGUGCGCGCUUUGUG -5' }\end{aligned}$

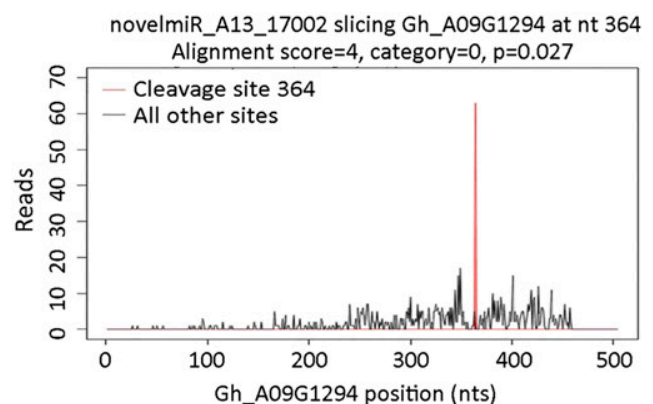

Gh_A09G1294: 5'- CCGGGUUCAGGUGCAAAUGCG -3'

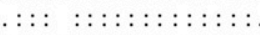

novelmiR_A13_17002: 3'- AUUCCACGUCCACGUUUACGU -5'

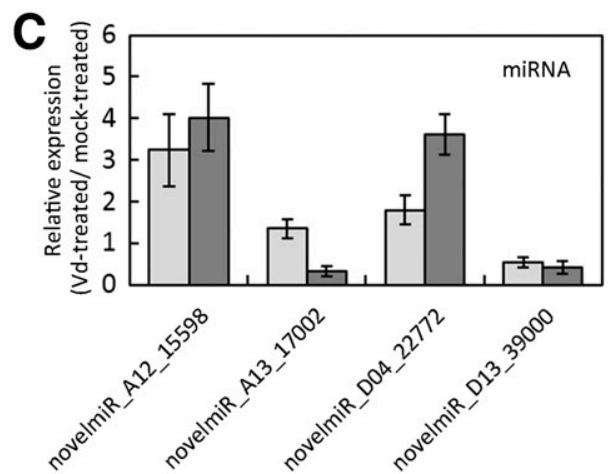

novelmiR_A12_15598 slicing Gh_A12G2506 at nt 511 Alignment score $=5$, category $=0, p=0.031$

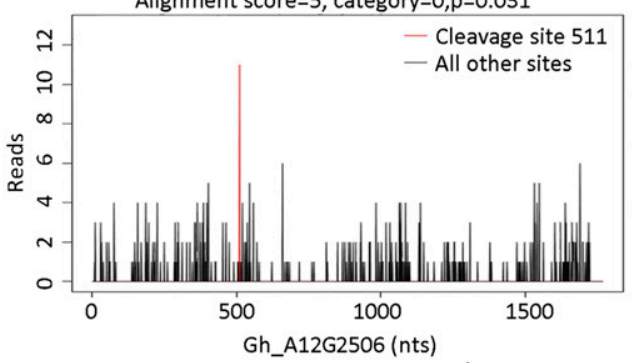

Gh_A12G2506: 5'- UUGUAGUCCUU'GAGGGAGAUU -3' novelmiR_A12_15598: 3'- GUCUUCGGGAACUCCCUCUCA -5'
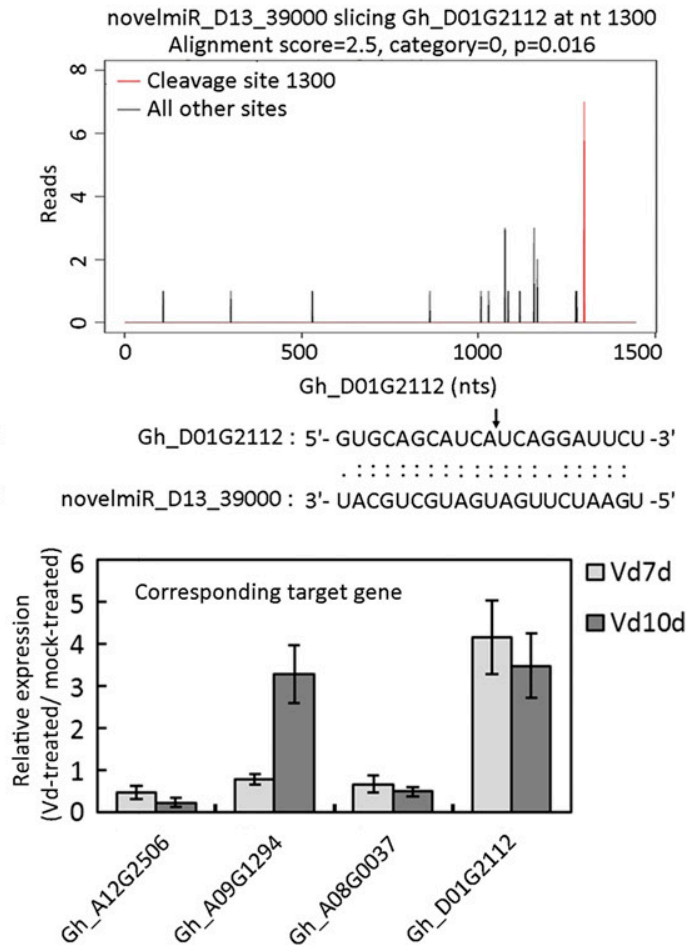

Fig. 3. Analyses of different expressions of the microRNAs (miRNAs) and target gene identification as well as expression authenticity. A, Heatmaps of differently expressed miRNAs in the 7- and 10-days postinoculation (dpi) samples compared with the control. The color bar represents the relative signal intensity values from red (upregulated) to blue (downregulated), indicating a range of 4 to -4 . B, Cotton miRNA and target alignment and its T-plot validated by degradome sequencing. The T-plots indicate the distribution of the degradome tags along the full length of the target mRNA sequencing. The black arrows indicate the cleavage sites of the target genes. C, Expression profiles of miRNAs and corresponding targets after Verticillium dahliae was inoculated by quantitative PCR. miRNAs and their corresponding targets detected from the roots infected with $V$. dahliae and mock-treated control at 7 and 10 dpi, respectively. Error bars represent the standard deviation of three biological replicates. Vd7d and Vd10d represent 7 and 10 days after $V$. dahliae infection. 
qPCR. Four different expression level miRNAs (Fig. 3A) and their corresponding target genes were chosen to analyze the expression levels in treated roots and the control. The expression levels of novel miR_A12_15598, novel miR_D04_22772, miR_D13_39000, and miR_A13_17002 in the 7- and 10-dpi treatments compared with the mock control were in line with the results of the sRNA sequencing in $V$. dahliae treatment (Fig. 3C; Supplementary Table S4). The expression levels of corresponding target genes at the 7- and 10-dpi timepoints compared with the mock control showed some contrasting trends with the expression profiles of the four miRNAs, indicating that these miRNAs may reduce the mRNA levels of the corresponding target genes through posttranscription regulation (Fig. 3C).

\section{A ghr-miR477 posttranscriptionally regulating GhCBP60A expression.}

Based on the degradome and sRNA sequencing, we found a novel miRNA, miR_A12_15598, which showed significant differentiate expression at 7 and $10 \mathrm{dpi}$, as compared with the control. The target gene of this novel miRNA was identified, in our degradome data, as CBP60A-like (CaM-binding protein 60A-like), which participates in plant defense against pathogens. Thereby, a miR_A12_15598 and CBP60A-like module was picked to further study their defense function in cotton. Homologous search revealed that the mature sequence of the novel miRNA is the same as nta-miR477a (MiRBase number MIMAT0024721) in Nicotiana tabacum, designated ghrmiR477. The secondary structure analysis by the mfold Web Server predicted a well-developed hairpin structure in the precursor of ghr-miR477 sequences (Supplementary Fig. S7). A phylogenetic tree shows that target gene coding protein CBP60A-like is clustered with AtCBP60a (51.22\% identification) and is designated GhCBP60A (Fig. 4A).

When ghr-miR477 matched the target gene GhCBP60A, the cleavage site was identified, as shown in the T-plots, located at the $511 \mathrm{nt}$ of $G h C B P 60 A$ mRNA and cleaved between the C and $\mathrm{U}$ bond (Fig. 3B). To confirm this cleavage site, two specific forward primers were designed, which were located on both sides of the cleavage site (Fig. 4B), and qPCR analysis was conducted. The amplification effectives of two primers were similar through amplifying analysis of genome DNA. As shown in Figure $4 \mathrm{~B}$, the amounts of the FD-amplified fragment downstream of the cleavage site were approximately 1.9-fold higher than the FU-amplified fragment containing the cleavage site. To further verify the cleavage sites of GhCBP60A mRNA by ghr-miR477, we performed a 5'-RNA ligase mediated-rapid amplification of cDNA ends ( $5^{\prime}$ RLM-RACE) analysis. Nine of the 10 clones showed that $G h C B P 60 A$ can be cleaved at the $511 \mathrm{nt}$ of $G$ CBP60A mRNA through sequencing analysis (Fig. 4C).

To confirm the ghr-miR477 function in cleaving its target sequence in vivo, a GhCBP60A:GUS reporter fusion protein was analyzed by the Agrobacterium tumefaciens-mediated cotransformation technology in Nicotiana benthamiana. The precursor of ghr-miR477 was isolated and the vector pBI121miR477 was constructed as an effector. The GhCBP60Aencoding sequence and its corresponding mutant sequence were respectively fused into the upstream of the $\beta$-glucuronidase (GUS) gene in the plant expression vector $\mathrm{pBI} 121$, generating pBI121-CBP60A:GUS and pBI121-CBP60A ${ }^{\mathrm{mu}}$ :GUS as reporters. As shown in Figure 4D, the leaves injected with GV3101 containing only pBI121 or pBI121-CBP60A:GUS exhibited a similar blue intensity in the infiltrated site, by GUS histochemical staining. When the leaves were infiltrated equally with pBI121-miR477 and pBI121-CBP60A:GUS, the blue spot was absent at the injection site, while the leaves coinfiltrated with pBI121-miR477 and pBI121-CBP60A ${ }^{\mathrm{mu}}$ : GUS showed a similar blue intensity to that with pBI121CBP60A:GUS (Fig. 4D). Compatible with GUS staining, a quantitative assay of the GUS activity showed consistent results in the extracted total protein from the infiltrated sites of the leaf through 4-methylumbelliferone (4-MU) analysis (Fig. 4E). The result of the GUS fusion protein reporter showed that ghr-miR477 could cleave $G h C B P 60 A$ by a posttranscriptional process.

To explore the role of the miR477-CBP60A module in the plant response to fungal infection, the pathogen-responsive expression profile of ghr-miR477 and GhCBP60A was analyzed. The results showed that the accumulation of ghrmiR477 increased at 7, 10, and 13 dpi in roots inoculated with pathogen compared with the mock treatments (Fig. 4F). And the GhCBP60A transcript level significantly decreased in the roots of plants challenged with $V$. dahliae at 10 and 13 dpi (Fig. 4F). These data confirmed that the ghr-miR477 negatively regulated GhCBP60A expression, particularly in the plants inoculated with $V$. dahliae.

\section{ghr-miR477 silencing improves plant resistance to $V$. dahliae.}

To determine the function of ghr-miR477 in plant defense, miRNA target mimicry technology was employed, which has been successfully used to suppress miRNA accumulation in vivo (Sha et al. 2014; Yan et al. 2012). We used the virus-based miRNA silencing (VbMS) strategy to generate ghr-miR477silenced plants. The pTRV2e-STTM477 vector, which contains two imperfect binding sites for ghr-miR477 separated by a 48-nt spacer, was constructed. The G. hirsutum phytoene desaturase $(G h P D S)$ gene was well-silenced, resulting in a photobleaching phenotype (Supplementary Fig. S8), indicating that the tobacco rattle virus (TRV) virus-induced gene silencing (VIGS) system is feasible in the cotton plant. Compared with the control plants inoculated with the empty vector (TRV:00), the abundance of ghr-miR477 transcripts was significantly reduced by approximately $35 \%$ in the TRV:STTM477 cotton plants (Fig. $5 \mathrm{~A})$. The GhCBP60A expression level showed an increase of approximately 1.6-fold compared with the $T R V$ :00 plants (Fig. $5 \mathrm{~A})$, suggesting that we successfully knocked down ghrmiR477 expression in the TRV:STTM477 plants by overexpressing a small tandem target mimic (STTM), using the VbMS. TRV:STTM477 plants and the control were then infected with $10^{6} \mathrm{~V}$. dahliae conidia. After $21 \mathrm{dpi}$, the $T R V$ : STTM477 plants showed more serious disease symptoms than the control, with obvious necrotic and wilting leaves and stunted growth (Fig. 5B). A fungal recovery assay showed that there were more fungal colonies in the TRV:STTM477 plants than in the TRV:00 plants (Fig. 5C). The disease index (DI) value in the TRV:STTM477 plants was significantly higher than that of the control, showing DI values of 74 versus 56 (Fig. 5D). Consistent with these results, the rate of diseased plants and fungal biomass in the TRV:STTM477 plants was significantly higher, 1.8-fold and 3.6-fold higher compared with the control, respectively (Fig. 5E and F).

\section{The miR477-CBP60A module regulates plant defense to $V$. dahlia.}

To clarify the roles of the miR477-CBP60A module in plant resistance to $V$. dahliae, the $G h C B P 60 A$ gene was knocked down by a TRV-mediated VIGS system. When the PDSsilenced plant leaves became chlorotic, we started to examine GhCBP60A expression levels of the plants injected with agrobacterium containing the TRV:GhCBP60A vector. According to a qPCR analysis, GhCBP60A expression levels in the $C B P 60 A$-silenced leaves significantly decreased to approximately $55 \%$ of the control (TRV:00 plants) (Fig. 5A). To 

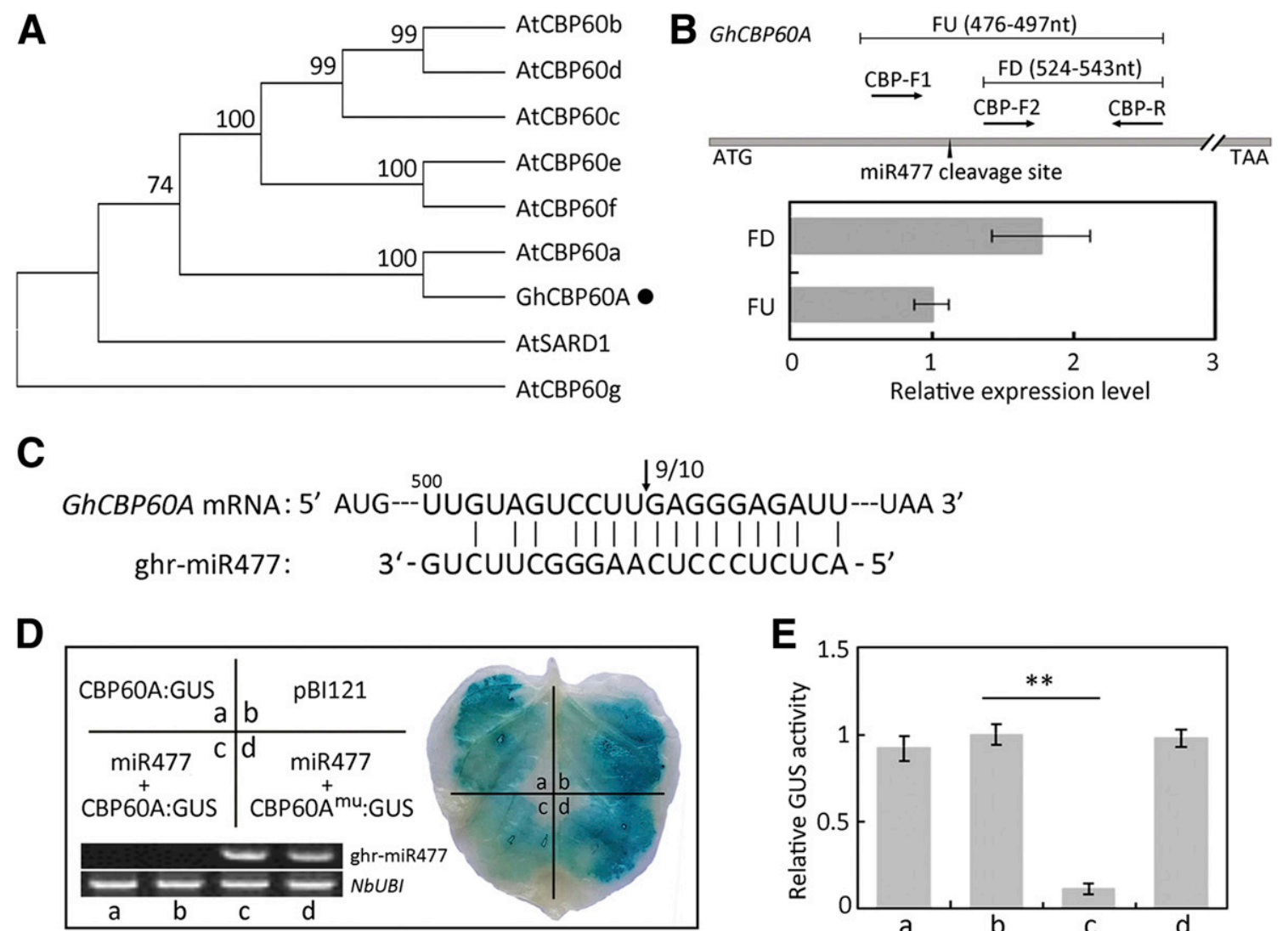

GGGAGAUU---UAA 3'

ACCCUCUCA - 5'

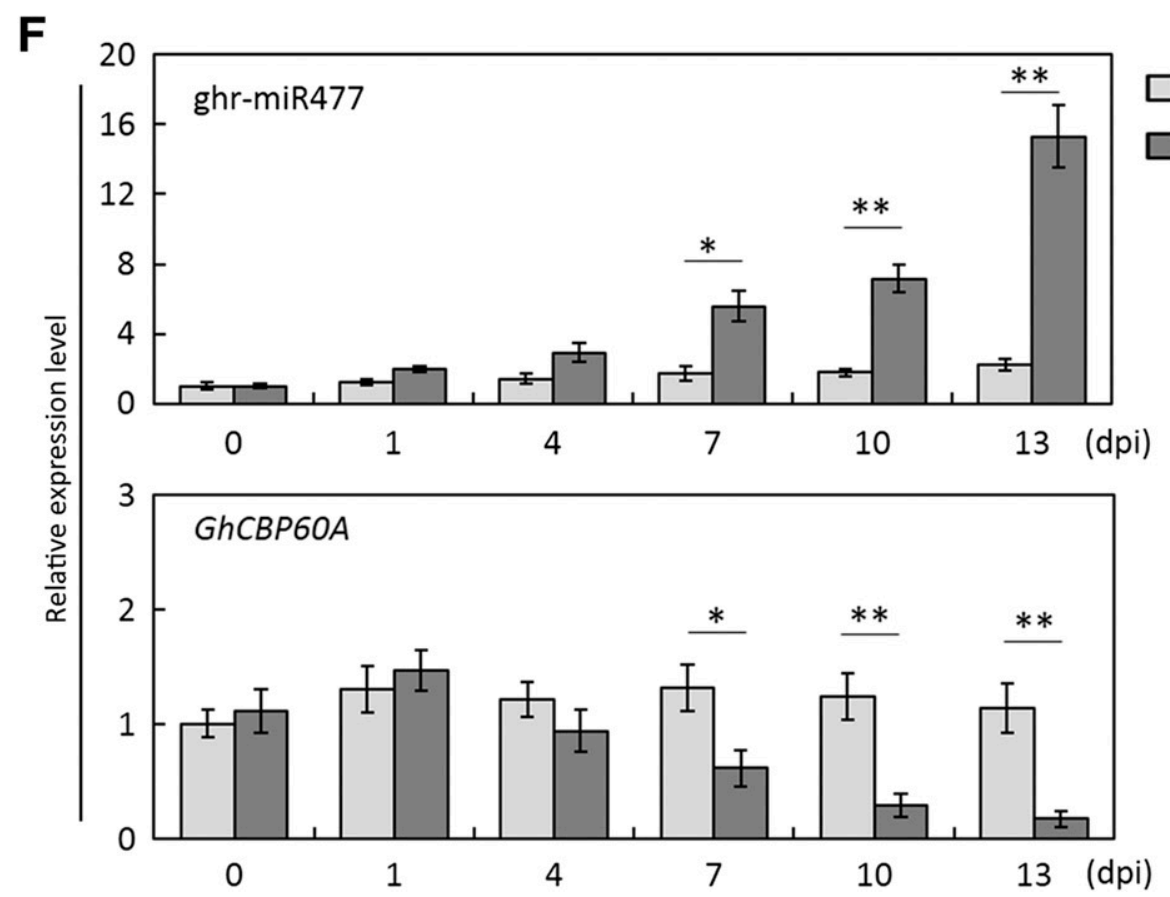

Fig. 4. ghr-miR477 regulates GhCBP60A expression by a posttranscriptional process. A, Phylogenetic tree analysis of GhCBP60A and AtCBPs. The neighborjoining method of MEGA (version 5.2) was used. Bootstrap analyses were computed with 1,000 replicates. B, Primer design outline and quantitative PCR analysis of the GhCBP60A mRNA transcripts associated with the ghr-miR477 cleavage. FD and FU = the PCR-amplified production locating downstream of the cleavage site and containing the cleavage site, respectively. The value of the FU-amplified fragment was arbitrarily designated as 1 . Error bars represent the standard deviation (SD) of three biological replicates. C, The cleavage sites of GhCBP60A mRNA were determined by the 5' RNA ligase mediated rapid amplification of cDNA ends (RACE) method. An arrow indicates the $5^{\prime}$ terminus of microRNA-guided cleavage products, as identified by $5^{\prime}$-RACE, with the frequency of clones (9/10) also shown. D, $\beta$-Glucuronidase (GUS) staining of infiltrated sites of the leaf with different vectors. E, Quantitative analysis of GUS activity illustrated in D, using a 4-methylumbelliferone assay. The value of GUS activity in the pBI121 was arbitrarily designated as 1 . F, Accumulation of ghrmiR477 and GhCBP60A in the time course of cotton roots infected with Verticillium dahliae. The value of ghr-miR477 and GhCBP60A under mock treatment at 0 days postinoculation (dpi) was arbitrarily designated as 1 . Error bars represent the SD of three biological replicates in E and F, asterisks indicate significant differences $(* P<0.05$, ** $P<0.01)$. 
evaluate GhCBP60A function in resistance to this fungus, the GhCBP60A-silenced plants and the control were infected with $V$. dahliae. At $21 \mathrm{dpi}$, the control showed normal disease symptoms with wilting, yellowing leaves, and stunted growth, while the $C B P 60 A$-silenced plants exhibited obvious resistance to this fungus (Fig. 5B). A fungal recovery assay showed that there were more fungal colonies in the control than in the CBP60Asilenced plants (Fig. 5C). The DI value, rate of diseased plants, and fungal biomass in the $G h C B P 60 A$-silenced plants were significantly lower than that in the control (Fig. 5D, E, and F). The results showed that GhCBP60A is possibly a negative regulator to increase plant resistance to $V$. dahliae.
The miR477-CBP60A module regulates $\mathrm{SA}$ biosynthesis to mediate plant defense.

Previous study had reported that CBPs participate in plant disease resistance involving a SA signal pathway (Truman et al. 2013). As GhCBP60A-silenced plants are more resistant to $V$. dahliae while ghr-miR477-silenced plants are more susceptible, we hypothesized that the SA level might be higher in GhCBP60A-silenced plants compared with the control. To test this, we measured SA levels in the TRV:00, TRV:STTM477, and TRV:CBP60A cotton plants at 4, 7, and $10 \mathrm{dpi}$. The SA levels at these three timepoints after 3 dpi were similar. As shown in Figure 6A, average SA levels were elevated in the $T R V$ :

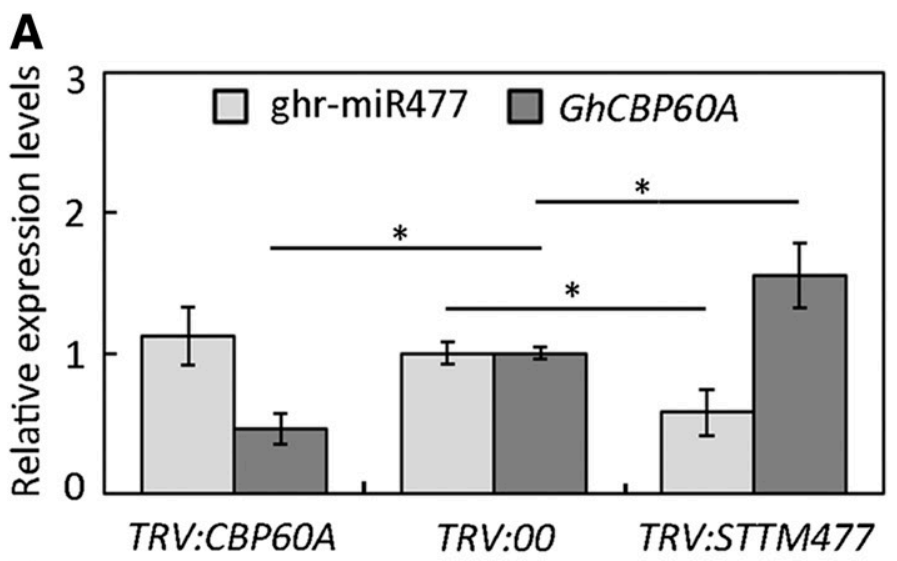

B

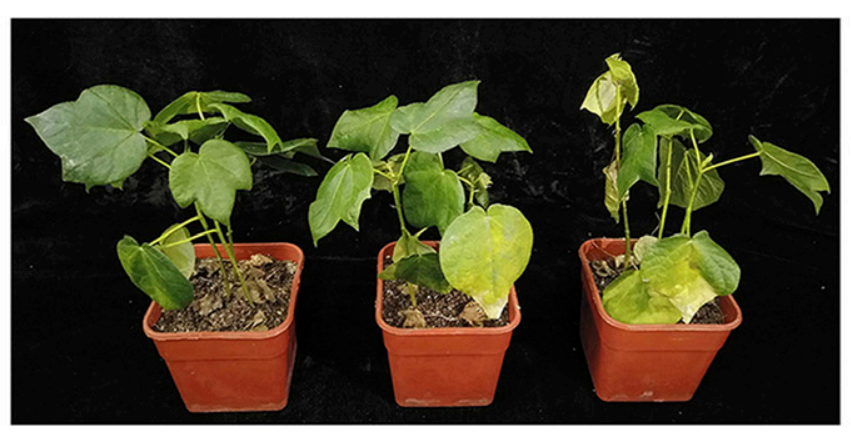

E

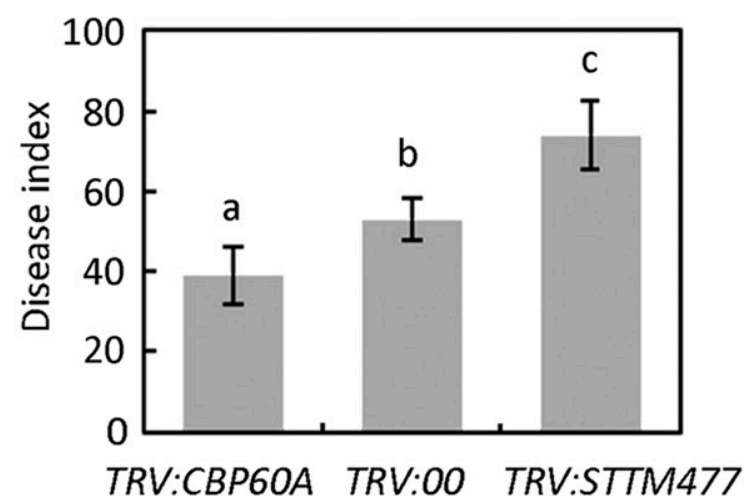

TRV:CBP6OA TRV:00 TRV:STTM477

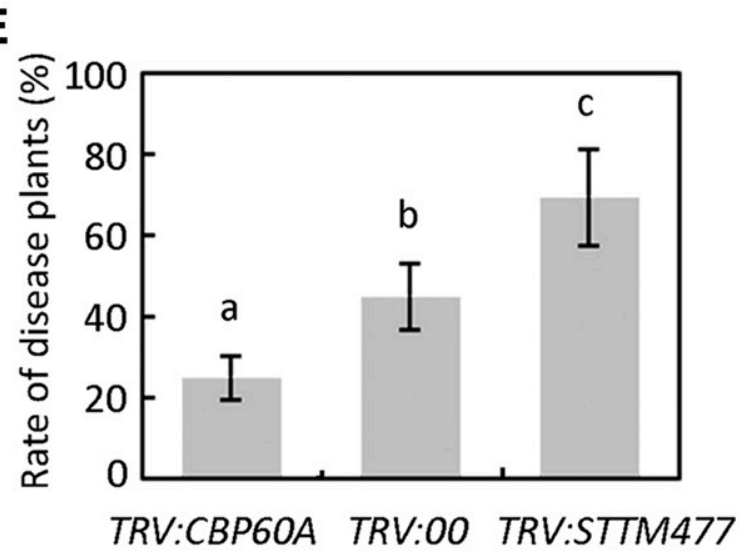

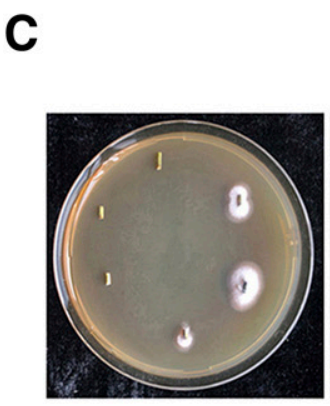

TRV:CBP60A

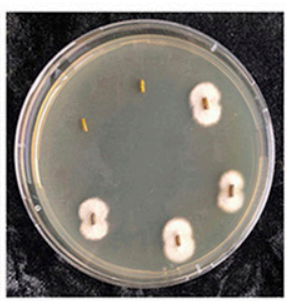

TRV:00

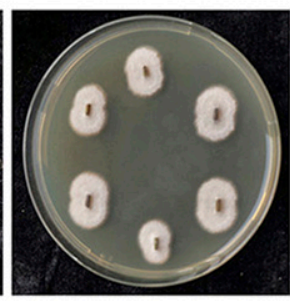

TRV:STTM477

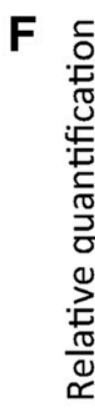

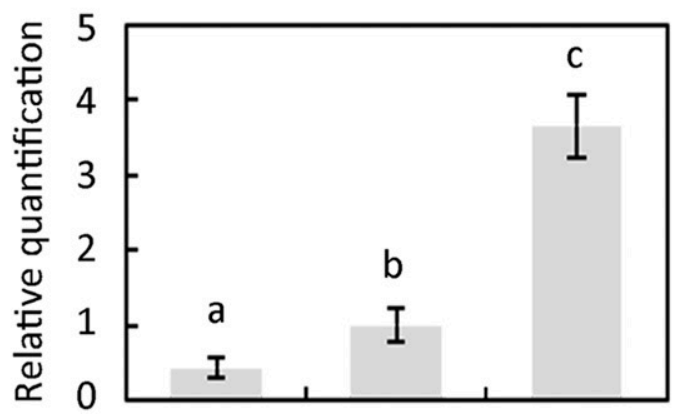

$T R V: C B P 60 A$

TRV:00 TRV:STTM477

Fig. 5. The functional dissection of the miR477-CBP60A module in defense against Verticillium dahlia. A, Relative expression levels of ghr-miR477 and GhCBP60A in TRV:STTM477 and TRV:GhCBP60A plants compared with TRV:00 plants. The value of ghr-miR477 and GhCBP60A in TRV:00 cotton plants was arbitrarily designated as 1 . Error bars represent the standard deviation (SD) of three biological replicates, and asterisks indicate significant differences, as determined by the Student's $t$ test (an asterisk indicates $P<0.05$ ). B, Disease symptom phenotypes of the ghr-miR477-silenced and $G h C B P 60 A$-silenced plants inoculated with $V$. dahliae. C, Fungal recovery assay. The experiment was performed using stem sections from cotton plants at 21 days postinoculation (dpi) placed on potato dextrose agar media. Photos were taken 5 days after plating. D, Disease index of the silenced plants at 21 dpi. E, The rate of diseased plants in TRV:00, TRV:STTM477, and TRV:CBP60A plants. F, Relative quantification of the fungal biomass in infected cotton stems. Quantitative PCR analysis was conducted to compare the DNA contents between the TUB1 gene of $V$. dahliae and the ACT4 gene of cotton. Error bars in D, E, and F represent the SD of three biological replicates and different letters indicate significant differences $(P<0.05)$ based on Tukey's honestly significant differences test. 
CBP60A plants, while they were lower in the TRV:STTM477 plants, indicating that CBP60A decreased the SA accumulation in the plant cell.

A previous report showed that Arabidopsis CBP60a is a negative regulator of plant immunity involving ICS1 and other defense responses (Truman et al. 2013). To confirm whether GhCBP60A is associated with expression of ICS1 and PATHOGENESIS RELATED 1 ( $P R 1$, a defense-related gene) in cotton plants, qPCR analysis was carried out. The expression of GhICS1 and GhPRl was significantly elevated in the TRV: $C B P 60 A$ plants after $V$. dahliae inoculation compared with the control (Fig. 6B). However, GhICS1 and GhPRI showed decreasing expression with $V$. dahliae inoculation in the $T R V$ : STTM477 plants (Fig. 6B). The results indicated that GhCBP60A may repress SA biosynthesis, which negatively regulates cotton plant defense to $V$. dahliae.

\section{DISCUSSION}

miRNAs participate in plant resistance to pathogens through posttranscriptional regulation of target gene expression. The response of the cotton plants to $V$. dahliae infestation may be divided into early and later stages including the pathogen localizing in the surface of roots and entering their interior. Previous reports involving sRNA sequencing focused only on the early response of the plant to $V$. dahliae infection, which in general reacts before $48 \mathrm{~h}$ after inoculation. However, there are few reports about the later response of the plant to this fungus. In this study, we constructed 7- and 10-day V. dahliae-infected root sRNA and identified many known and novel miRNAs. miRNAs differentially expressed at 7 and 10 dpi were analyzed compared with the mock control combined with degradome sequencing. The results showed that 71 known miRNAs and 378 novel miRNAs were identified from 7- and 10-dpi and control treatments, with two biological repeats, and 34 differentially expressed miRNAs in both 7- and 10-dpi treatments were analyzed compared with the control. More importantly, a miR477-CBP60A module was selected to represent the miRNAs and corresponding targets, to perform functional dissection of plant defense against $V$. dahliae.

We identified 378 novel miRNAs from the six sRNA libraries, including the 7- and 10-dpi and the mock-treated control, with two repeats. However, previous studies showed that significantly fewer novel miRNAs were identified at 12, 24, or $48 \mathrm{~h}$ after inoculation (He et al. 2014; Yin et al. 2012; Zhang et al. 2015a). For instance, 14 novel miRNAs were identified from Verticillium-inoculated cotton roots at 12 and
$24 \mathrm{~h}$ (Yin et al. 2012), 37 novel miRNAs were identified from two sRNAs of cotton seedlings inoculated with $V$. dahliae at 24 and $48 \mathrm{~h}$ (He et al. 2014), and 58 novel miRNAs were identified from sRNA sequencing of the cotton roots inoculated with $V$. dahliae at $24 \mathrm{~h}$ (Zhang et al. 2015a). Thus, in this study, more novel miRNAs were identified that may participate in the resistance of the plant to $V$. dahliae at later stages of fungal infection. Moreover, we found that various differentially expressed miRNAs emerged in miRNA data for the early induction stage and that of later induction stages. For instance, the differentially expressed ghr-miR2950 was reported in data of two miRNA from the early stages of $V$. dahliae infection (He et al. 2014; Yin et al. 2012), but it did not show differential expression in the miRNA data of a later stage of $V$. dahliae infection. However, there were five differential expression miRNAs, including ghr-miR398, ghr-miR479, ghr-miR7507, ghr-miR7497, and ghr-miR7499, in our miRNA data of the late induction stage that were not reported in the previous miRNA data of the early induction stage. Thereby, the five miRNAs possibly play roles in plant response to fungal infection at the late stage, worthy of further research.

At 7 and $10 \mathrm{dpi}$, the plants should have a stronger response to fungal infection, possibly because $V$. dahliae has localized in the xylem vessels, unlike the pathogen surface-induced response at the early stage of inoculation. The target genes of the differentially expressed miRNAs predominantly participated in many GO classifications, including the oxidation-reduction process and stress response (Supplementary Table S10). KEGG analysis focused on 10 pathways, and the significantly enriched pathways include terpenoid backbone biosynthesis, carotenoid biosynthesis, and the spliceosome (Supplementary Table S11). However, in the literature associated with fungal surfaceinduced miRNAs, there is no data on the GO and KEGG analyses of target genes (He et al. 2014; Yin et al. 2012; Zhang et al. 2015a). These results supposed that the internal induced response of the plant by $V$. dahliae may be stronger, possibly by modulating miRNA expression.

SA, a phytohormone, is an essential systemic signal for plant resistance against pathogens (Vlot et al. 2009). When plants are attacked by pathogens, their SA level rapidly increases in infected sites to activate the expression of downstream defenserelated genes and systemic acquired resistance is induced in noninfected sites through the SA signal pathway, resulting in plant resistance (Spoel and Dong 2012; Klessig et al. 2018). In Arabidopsis, CBP60a, CBP60g, and SARD1 in eight CBP60 members participate in plant innate immunity under the presence of $\mathrm{CaM}$ and $\mathrm{Ca}^{2+}$, which promote or repress the basal
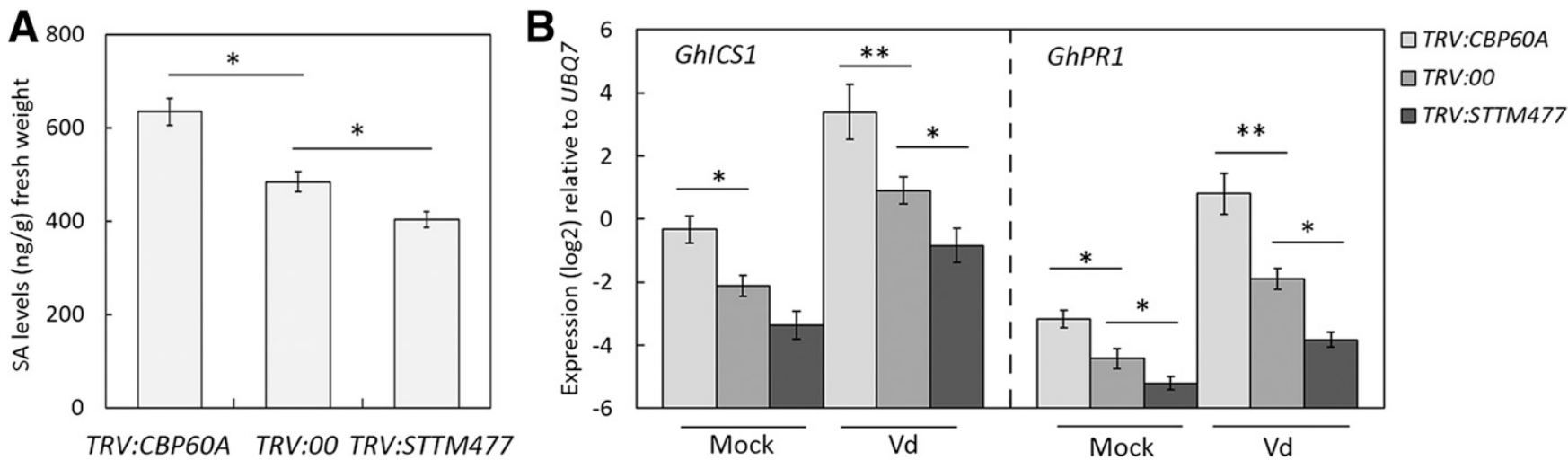

Fig. 6. The miR477-CBP60A module elevated salicylic acid (SA) level in cotton. A, SA accumulation in cotton plants inoculated with Verticillium dahliae. B, Quantitative PCR analysis of the SA signal gene in GhCBP60A-silenced plants treated with $V$. dahliae in cotton roots. Error bars represent the standard deviation of three biological replicates, and asterisks indicate significant differences, as determined by the Student's $t$ test (one asterisk $(*)$ indicates $P<0.05$, two (**) $P<0.01)$. 
expression of genes (Sun et al. 2015). Generally, CBP60a represses immunity and CBP60g and SARD1 have low activity in the absence of pathogen. When pathogens attack the plants, the CBP60a may be inhibited, but the repression of CBP60g and SARD1 can be removed in immunity, resulting in promotion of SA synthesis to increase plant defense (Truman et al. 2013; Wang et al. 2011). The cbp60a mutants showed that it is epistatic to CBP60g in response to pathogen infection (Truman et al. 2013). Here, we measured the SA level of plants at the later induction stage of fungal infection and found that the SA contents were accumulated in $G h C B P 60 A$-silencing plants and were decreased in TRV:STTM477 plants, indicating that SA plays important roles in plant resistance at the later induction stage. However, what modulates the CBP60a activity remains unclear in plant defense. In this study, we found that a novel cotton miRNA, ghr-miR477, can mediate the GhCBP60A activity through posttranscriptional regulation. Collectively, in the presence of pathogens, miR477-CBP60A can finely tune SA biosynthesis and enhance cotton CBP60g and SARD1 activity, leading to an increase of plant defense.

Previous reports involving the sRNA sequencing of cotton plants in response to $V$. dahliae focused on the early induction stage, typically 12 to $48 \mathrm{~h}$ after inoculation when the fungus localized on the surface of the roots. We acquired the sRNA profiles of the plant response to $V$. dahlia focused on the later induction stage when fungi had localized in the interior root tissues. We identified 71 known miRNAs and 378 novel miRNAs from 7 and $10 \mathrm{dpi}$ and the control libraries and investigated their target categories using GO and KEGG analyses. Thirty-four of these miRNAs showed significantly different expression in the two infected treatments compared with the control. More importantly, according to the degradome and sRNA sequencing, we selected the miR477-CBP60A module as a representative to evaluate the function of the miRNAs in the response of the plant to the fungus through posttranscriptional regulation. The results showed that miR477-CBP60A mediates SA biosynthesis and other resistance genes to participate in cotton plant resistance.

Based on sRNA and degradome sequencing, we chose the cotton miR477-CBP60A module as a representative to analyze the function of the miRNAs coupling with their target genes in the resistance of the plant to $V$. dahliae. Our results showed that ghr-miR477 silencing increased the susceptibility of the plant to this fungus, while GhCBP60A knockdown elevated the resistance of the plants. Therefore, the miR477-CBP60A module participates in plant defense.

\section{MATERIALS AND METHODS}

\section{Plant growth condition and treatment.}

G. hirsutum cv. Jihe713 was donated by X. Luo from the Institute of Cotton Research, Shanxi Academy of Agricultural Science. Seedlings grew in a greenhouse at $28^{\circ} \mathrm{C}$ under a $16-\mathrm{h}$ light and 8-h dark photoperiod.

To conduct high-throughput sRNA and degradome sequencing at the two-leaf stage, cotton plants under hydroponic conditions were inoculated with $V$. dahliae V991. The 7- and 10-dpi roots as well as the mock-treated control were harvested. The inoculated roots were treated ultrasonically $(30 \mathrm{~s}$ with a gap of $30 \mathrm{~s}$, repeated five times) to remove the fungal hyphae and conidia on their surface. Three types of samples were immediately frozen in liquid nitrogen and were stored at $-80^{\circ} \mathrm{C}$ prior to RNA isolation for highthroughput sRNA sequencing, degradome sequencing, and qPCR analysis. The same experiment was repeated twice.

$N$. benthamiana plants were grown in the greenhouse under a 16-h light and 8-h dark photoperiod at $23^{\circ} \mathrm{C}$ for gene transient expression analyses.
Fungal cultivation and inoculation.

V. dahliae V991, a strongly pathogenic defoliating isolate, was cultured on potato dextrose agar (PDA) media for a week at $25^{\circ} \mathrm{C}$. The mycelia were transferred into Czapek-Dox media for a week at $25^{\circ} \mathrm{C}$, with shaking $(180 \mathrm{rpm})$ to collect the conidia. For $V$. dahliae infection, the roots of cotton plants were dipped with a conidial suspension $\left(10^{6}\right.$ conidia per milliliter $)$ for $50 \mathrm{~min}$. Subsequently, the plants were transferred into fresh, steamsterilized water for culture or were planted into the pot with soil.

\section{RNA extraction, qPCR, and quantitative} semi-reverse transcription (semi-qRT)-PCR analysis.

Total RNA was isolated from cotton samples using the PureLink plant RNA reagent (Life Technologies) according to the manufacturer instructions. First-strand cDNA was synthesized using an EasyScript first-strand cDNA synthesis supermix (TransGen). miRNA first-strand cDNA synthesis, qPCR analysis, semi-qRT-PCR, and primer design were conducted as described by Varkonyi-Gasic et al. (2007). The qPCR experiment was performed using a TransStart top green qPCR supermix kit (TransGen) in a $20-\mu$ reaction volume on a CFX96TM real-time detection system (Bio-Rad Laboratories, Inc.). The $G$. hirsutum $5.8 S$ gene was used as an internal control for miRNA. The $U B Q 7$ gene from $G$. hirsutum was used as an internal control. The $2^{-\Delta \Delta \mathrm{CT}}$ cycle threshold method was used to determine the relative expression levels of the miRNAs and target genes.

Fungal biomass quantification with qPCR techniques was performed as described previously (Wang et al. 2017b). The primer pairs to detect the $V$. dahliae TUBI gene and the cotton ACT4 gene were used for qPCR. The primers used for qPCR and semi-qRT-PCR are listed in Supplementary Table S12.

\section{Construction of the sRNA and degradome libraries.}

The cotton sRNA libraries were constructed, using a NEB Next, ultra sRNA sample library prep kit (Illumina), according to the manufacturer instructions. sRNA was purified from $1.5 \mu \mathrm{g}$ of the total RNA by using the sRNA sample pre kit and were ligated first to a 5' RNA adaptor and then to a $3^{\prime}$ RNA adaptor, using T4 RNA Ligase 1 and T4 RNA Ligase 2 (truncated). RT synthesis cDNA was purified by polyacrylamide gel electrophoresis as the sRNA library. The sRNA libraries were subjected to high-throughput sequencing with HiSeq2500 (Illumina), with a read length of single-end $50 \mathrm{nt}$ at the Biomarker Technologies Company in Beijing.

The degradome libraries were constructed as previously described (German et al. 2008). The equal-concentation total RNAs from root samples from the 7- and 10-dpi and mock treatments were extracted mixed, as a degradome libraries analysis. Briefly, a 5' RNA adaptor with a MmeI recognition site at the $3^{\prime}$ end was ligated to the resulting 42-bp fragments, consisting of a free phosphate at the $5^{\prime}$ end, followed by RT to cDNA. After PCR amplification, they were digested by the enzyme MmeI and were ligated to an Illumina $3^{\prime}$ TruSeq adaptor, followed by PCR amplification with a library-specific index primer and a common $5^{\prime}$ primer for multiplex sequencing, were gel-purified, and were subjected to sequencing by synthesis using HiSeq2500 (Illumina) at the Biomarker Technologies Company in Beijing.

\section{Identification and analysis of known and novel miRNAs.}

Raw sequences obtained from the six sRNA libraries were first cleaned by filtering out low-quality tags, poly(A) tags, and tags with $3^{\prime}$ adaptor nulls, insert nulls, 5' adaptor contaminants, or those smaller than $18 \mathrm{nt}$. The clean reads of twice repeats were integrated, and clean read number for each treatment was calculated to obtain a mean value. Using Bowtie tools soft 
(Langmead et al. 2009), rRNA, tRNA, snoRNA, and other noncoding RNA and repeats were filtered from the clean reads, respectively, with the Silva, GtRNAdb, Rfam, and Repbase database sequence alignments. The remaining sequences from 18 to $30 \mathrm{nt}$ long were used for miRDeep2 and miRBase to identify conserved miRNAs and novel $5^{\prime}$ - and $3{ }^{\prime}$-derived miRNAs (Friedländer et al. 2012; Zhang et al. 2015b). Only the sequences that were $\leq 2$ mismatches with known miRNAs were considered as conserved miRNAs. Otherwise, the reads were defined as nonconserved reads. Unannotated reads were used to predict novel miRNAs based on the characteristic hairpin structure of the microRNA precursors using miRDeep2 (Friedländer et al. 2012). The miRDeep2 software was used to sequence the unannotated reads with the reference genome (TM-1 v1.1) to obtain the positional information on the reference cotton genome, which is mapped reads.

\section{Differential expression analysis.}

The mean reads of each treatment were normalized by TPM, normalized expression $=($ actual miRNA count $/$ total count of clean reads) $\times 1,000,000$ (Fahlgren et al. 2007) . Differential expression analysis of the inoculated root treatments compared with the control was performed using the DESeq $\mathrm{R}$ package (Anders and Huber 2010). To investigate differentially expressed miRNAs between the treatments and the control, the fold change of each identified miRNA was calculated as the ratio of read counts in the treatments to the read counts in the control, followed by the transformation of $\log _{2}$. A value of the $\log _{2}$ ratio $\geq 1$ or $\leq-1$, indicating the ratio of fold change values for the treatments and control, were considered to be significantly differentially expressed. To show the differential expression profiles, heatmaps and clusters were constructed for the miRNAs, using ImageGP.

\section{Identification of miRNAs targets by degradome sequencing.}

The sequences of clean full-length reads collated from the degradome sequencing were used for subsequent analysis after removing low-quality sequences and adaptors. There were no mismatches allowed on nt 10 and 11 of the mature miRNAs, where the splice site on miRNA targets generally occurs during degradome analysis. A potential miRNA target with a $P$ value of $<0.05$, by PAREsnip software, was retained, and T-plot figures were drawn. All the target sequences were categorized into five classes based on the abundance of the degradome tags indicating miRNA-mediated cleavage. Category 0-4 was determined as previously described (Liu et al. 2014).

\section{Function enrichment analysis.}

The miRNA targets in the plants were predicted with TargetFinder software (Allen et al. 2005). GO enrichment analysis of the target genes corresponding to the miRNAs and differentially expressed miRNAs was implemented with GOseq R packages based on Wallenius noncentral hyper-geometric distribution (Ashburner et al. 2000).

KEGG is a database resource to understand high-level functions and utilities of the biological system, such as the cell, the organism, and the ecosystem, from molecular-level information, especially large-scale molecular datasets generated by genome sequencing and other high-throughput experimental technologies (Kanehisa et al. 2004). We used KOBAS software (Mao et al. 2005) to test the statistical enrichment of differential expression genes in KEGG pathways.

\section{Phylogenetic analysis.}

The $C B P$ genes in this study were retrieved from the The Arabidopsis Information Resource database and were aligned with the Clustal $\mathrm{X}$ program. Neighbor-joining phylogenetic trees were constructed in MEGA 5.2 with 1,000 bootstrap replicas (Tamura et al. 2011).

\section{Gene isolation and vector construction.}

GhCBP60A was isolated and mutated by PCR methods. GhCBP60A and $G h C B P 60 A^{m u}$ were inserted into pBI121 resulting, respectively, in the pBI121-CBP60A:GUS and pBI121-CBP60A ${ }^{\mathrm{mu}}$ :GUS vectors. The ghr-miR477 precursor sequence was isolated and inserted into the pBI121 instead of the gus gene, constructed in pBI121-miR477.

For the VIGS analysis, TRV-based vectors, including pYL192, pYL156, and pTRV2e, were used in this study. TRV: PDS was employed as a positive control vector in the silenced plants reported by Pang et al. (2013). The construction of the TRV-related vectors was performed as described by Liu et al. (2004) and Sha et al. (2014). Briefly, a STTM sequence of ghrmiR477 containing two imperfect ghr-miR477 binding sites separated by a 48-bp spacer with the restriction enzyme sites $K p n I$ and $X m a I$ at the $5^{\prime}$ and $3^{\prime}$ ends, respectively, was designed and inserted into the pTRV2e vector to generate the TRV: STTM477 vector (Supplementary Table S12). A GhCBP60A fragment was isolated and inserted into pTRV2, and the resulting vector was designated TRV:CBP60A. All the plasmids were transformed into Agrobacterium tumefaciens GV3101 using electroporation. All the primers associated with vector construction are listed in Supplementary Table S12.

\section{Gene transient expression analysis in $N$. benthamiana leaves.}

Agrobacterium cells, grown overnight at $28^{\circ} \mathrm{C}$ in lysogeny broth media containing $50 \mu \mathrm{g}$ of kanamycin per milliliter, $50 \mu \mathrm{g}$ of gentamicin per milliliter, and $50 \mu \mathrm{g}$ of rifampicin per milliliter, were collected and resuspended in methyl methacrylate infiltration media. After $3 \mathrm{~h}$ of incubation, the suspensions were infiltrated into the $N$. benthamiana leaves using a $2-\mathrm{ml}$ needleless syringe. After $12 \mathrm{~h}$ of incubation in darkness, tobacco seedlings were transferred to the greenhouse for normal growth.

\section{GUS activity analysis.}

At $48 \mathrm{~h}$ after agroinfiltration, treated leaves were detached, and GUS staining analysis was performed as described by Jefferson et al. (1987). GUS activity was quantified by 4-MU testing methods as described by Jefferson et al. (1987).

\section{5' RLM-RACE.}

RNA was extracted from a mixture of two-week-old cotton seedlings. The GhCBP60A cleavage sites were validated through a GeneRacer kit (Thermo Fisher Scientific), according to the manufacturer protocols. The PCR products were gel-purified, were subcloned into pEasy vectors (TransGen), and were sequenced. The sequencing results were analyzed to map the cleavage sites. The gene-specific primers were designed by Premier 5.0 software (Supplementary Table S12).

\section{Development of VIGS plants.}

Agrobacterium culture and treatments were the same as the method of agrobacterium cotransformation in tobacco described above. Agrobacterium cells containing TRV:CBP60A or $T R V: S T T M 477$ were mixed with an equal amount of agrobacterium cells with pTRV1 (pYL192). The mixed agrobacterium cells were agroinoculated into the fully expanded cotyledons of cotton seedlings, using a sterile needleless syringe. After two weeks, the leaf of the VIGS plant was used to extract RNA for gene expression analysis. 


\section{Disease resistance analysis.}

The DI is an important parameter to assess plant resistance, calculated according to the following formula: $\mathrm{DI}=[(\Sigma$ disease grades $\times$ number of infected plants)/(total checked plants $\times 4$ )] $\times 100$. Seedlings were classified into five grades (grade $0,1,2$, 3 , and 4) based on the disease severity after $V$. dahliae infection, as described by Wang et al. (2004). The stems and roots of the infected plants were collected to analyze the fungal recovery potential. The samples were cut into many fragments and were placed on PDA in plates, which were incubated at $25^{\circ} \mathrm{C}$. After 5 days, the number of fragments with fungal hypha was recorded.

\section{SA measure.}

Fresh leaves of cotton plants inoculated with V. dahliae at 4, 7, and 10 dpi were harvested, were frozen in liquid nitrogen, and were stored at $-80^{\circ} \mathrm{C}$ until needed. Fifty milligrams of fresh weight were ground into powder and were extracted with methanol/water/formic acid (15:4:1, vol/vol/vol). The combined extracts were evaporated to dryness under nitrogen gas stream, were reconstituted in $80 \%$ methanol $(\mathrm{vol} / \mathrm{vol})$, and were filtrated (PTFE filters, $0.22 \mu \mathrm{m}$; Anpel) before liquid chromatographytandem mass spectrometry (LC-MS/MS) analysis. SA contents were detected by MetWare based on the AB Sciex QTRAP 6500 LC-MS/MS platform. Three replicates of each assay were performed.

\section{AUTHOR-RECOMMENDED INTERNET RESOURCES}

The Arabidopsis Information Resource database: https://www.arabidopsis.org ImageGP: http://www.ehbio.com/ImageGP/index.php/Home/Index/index.html MetWare: https://www.metware.cn

miRBase sequence download page: www.mirbase.org/ftp.shtml

\section{LITERATURE CITED}

Allen, E., Xie, Z., Gustafson, A. M., and Carrington, J. C. 2005. microRNAdirected phasing during trans-acting siRNA biogenesis in plants. Cell 121:207-221.

Anders, S., and Huber, W. 2010. Differential expression analysis for sequence count data. Genome Biol. 11:R106.

Ashburner, M., Ball, C. A., Blake, J. A., Botstein, D., Butler, H., Cherry, J. M., Davis, A. P., Dolinski, K., Dwight, S. S., Eppig, J. T., Harris, M. A., Hill, D. P., Issel-Tarver, L., Kasarskis, A., Lewis, S., Matese, J. C., Richardson, J. E., Ringwald, M., Rubin, G. M., and Sherlock, G. 2000. Gene ontology: Tool for the unification of biology. Nat. Genet. 25:25-29.

Bejarano-Alcázar, J., Blancolopez, M. A., Melerovara, J. M., and Jimenezdiaz, R. M. 1997. The influence of verticillium wilt epidemics on cotton yield in southern Spain. Plant Pathol. 46:168-178.

Bhat, R. G., and Subbarao, K. V. 1999. Host range specificity in Verticillium dahliae. Phytopathology 89:1218-1225.

Bouché, N., Yellin, A., Snedden, W. A., and Fromm, H. 2005. Plant-specific calmodulin-binding proteins. Annu. Rev. Plant Biol. 56:435-466.

Fahlgren, N., Howell, M. D., Kasschau, K. D., Chapman, E. J., Sullivan, C. M., Cumbie, J. S., Givan, S. A., Law, T. F., Grant, S. R., Dangl, J. L., and Carrington, J. C. 2007. High-throughput sequencing of Arabidopsis microRNAs: Evidence for frequent birth and death of MIRNA genes. PLoS One 2:e219.

Friedländer, M. R., Mackowiak, S. D., Li, N., Chen, W., and Rajewsky, N. 2012. miRDeep2 accurately identifies known and hundreds of novel microRNA genes in seven animal clades. Nucleic Acids Res. 40:37-52.

German, M. A., Pillay, M., Jeong, D. H., Hetawal, A., Luo, S., Janardhanan, P., Kannan, V., Rymarquis, L. A., Nobuta, K., German, R., De Paoli, E., Lu, C., Schroth, G., Meyers, B. C., and Green, P. J. 2008. Global identification of microRNA-target RNA pairs by parallel analysis of RNA ends. Nat. Biotechnol. 26:941-946.

He, X., Sun, Q., Jiang, H., Zhu, X., Mo, J., Long, L., Xiang, L., Xie, Y., Shi, Y., Yuan, Y., and Cai, Y. 2014. Identification of novel microRNAs in the Verticillium wilt-resistant upland cotton variety $\mathrm{KV}-1$ by highthroughput sequencing. Springerplus 3:564.

Jefferson, R. A., Kavanagh, T. A., and Bevan, M. W. 1987. GUS fusions: Beta-glucuronidase as a sensitive and versatile gene fusion marker in higher plants. EMBO J. 6:3901-3907.
Jones-Rhoades, M. W., Bartel, D. P., and Bartel, B. 2006. MicroRNAS and their regulatory roles in plants. Annu. Rev. Plant Biol. 57:19-53.

Kanehisa, M., Goto, S., Kawashima, S., Okuno, Y., and Hattori, M. 2004. The KEGG resource for deciphering the genome. Nucleic Acids Res. 32: D277-D280.

Khraiwesh, B., Zhu, J. K., and Zhu, J. 2012. Role of miRNAs and siRNAs in biotic and abiotic stress responses of plants. Biochim. Biophys. Acta 1819:137-148.

Klessig, D. F., Choi, H. W., and Dempsey, D. A. 2018. Systemic acquired resistance and salicylic acid: Past, present, and future. Mol. PlantMicrobe Interact 31:871-888.

Klosterman, S. J., Atallah, Z. K., Vallad, G. E., and Subbarao, K. V. 2009. Diversity, pathogenicity, and management of verticillium species. Annu. Rev. Phytopathol. 47:39-62.

Langmead, B., Trapnell, C., Pop, M., and Salzberg, S. L. 2009. Ultrafast and memory-efficient alignment of short DNA sequences to the human genome. Genome Biol. 10:R25.

Li, S., Liu, L., Zhuang, X., Yu, Y., Liu, X., Cui, X., Ji, L., Pan, Z., Cao, X., Mo, B., Zhang, F., Raikhel, N., Jiang, L., and Chen, X. 2013. MicroRNAs inhibit the translation of target mRNAs on the endoplasmic reticulum in Arabidopsis. Cell 153:562-574.

Li, Y., Lu, Y. G., Shi, Y., Wu, L., Xu, Y. J., Huang, F., Guo, X. Y., Zhang, Y., Fan, J., Zhao, J. Q., Zhang, H. Y., Xu, P. Z., Zhou, J. M., Wu, X. J., Wang, P. R., and Wang, W. M. 2014. Multiple rice microRNAs are involved in immunity against the blast fungus Magnaporthe oryzae. Plant Physiol. 164:1077-1092.

Liu, H., Qin, C., Chen, Z., Zuo, T., Yang, X., Zhou, H., Xu, M., Cao, S., Shen, Y., Lin, H., He, X., Zhang, Y., Li, L., Ding, H., Lübberstedt, T., Zhang, Z., and Pan, G. 2014. Identification of miRNAs and their target genes in developing maize ears by combined small RNA and degradome sequencing. BMC Genomics 15:25.

Liu, Y., Nakayama, N., Schiff, M., Litt, A., Irish, V. F., and Dinesh-Kumar, S. P. 2004. Virus induced gene silencing of a DEFICIENS ortholog in Nicotiana benthamiana. Plant Mol. Biol. 54:701-711.

Mao, X., Cai, T., Olyarchuk, J. G., and Wei, L. 2005. Automated genome annotation and pathway identification using the KEGG orthology (KO) as a controlled vocabulary. Bioinformatics 21:3787-3793.

Mi, S., Cai, T., Hu, Y., Chen, Y., Hodges, E., Ni, F., Wu, L., Li, S., Zhou, H., Long, C., Chen, S., Hannon, G. J., and Qi, Y. 2008. Sorting of small RNAs into Arabidopsis argonaute complexes is directed by the 5' terminal nucleotide. Cell 133:116-127.

Navarro, L., Dunoyer, P., Jay, F., Arnold, B., Dharmasiri, N., Estelle, M., Voinnet, O., and Jones, J. D. 2006. A plant miRNA contributes to antibacterial resistance by repressing auxin signaling. Science 312: 436-439.

Pang, J., Zhu, Y., Li, Q., Liu, J., Tian, Y., Liu, Y., and Wu, J. 2013. Development of agrobacterium-mediated virus-induced gene silencing and performance evaluation of four marker genes in Gossypium barbadense. PLoS One 8:e73211.

Rajagopalan, R., Vaucheret, H., Trejo, J., and Bartel, D. P. 2006. A diverse and evolutionarily fluid set of microRNAs in Arabidopsis thaliana. Genes Dev. 20:3407-3425.

Sha, A., Zhao, J., Yin, K., Tang, Y., Wang, Y., Wei, X., Hong, Y., and Liu, Y. 2014. Virus-based microRNA silencing in plants. Plant Physiol. 164: $36-47$.

Shriram, V., Kumar, V., Devarumath, R. M., Khare, T. S., and Wani, S. H. 2016. MicroRNAs as potential targets for abiotic stress tolerance in plants. Front. Plant Sci. 7:817.

Sink, K. C., and Grey, W. E. 1999. A root-injection method to assess verticillium wilt resistance of peppermint (Mentha $\times$ piperita L.) and its use in identifying resistant somaclones of cv. Black Mitcham. Euphytica 106:223-230.

Song, Q. X., Liu, Y. F., Hu, X. Y., Zhang, W. K., Ma, B., Chen, S. Y., and Zhang, J. S. 2011. Identification of miRNAs and their target genes in developing soybean seeds by deep sequencing. BMC Plant Biol. 11:5.

Spoel, S. H., and Dong, X. 2012. How do plants achieve immunity? Defence without specialized immune cells. Nat. Rev. Immunol. 12:89-100.

Sun, T., Zhang, Y., Li, Y., Zhang, Q., Ding, Y., and Zhang, Y. 2015. ChIPseq reveals broad roles of SARD1 and CBP60g in regulating plant immunity. Nat. Commun. 6:10159

Tamura, K., Peterson, D., Peterson, N., Stecher, G., Nei, M., and Kumar, S. 2011. MEGA5: Molecular evolutionary genetics analysis using maximum likelihood, evolutionary distance, and maximum parsimony methods. Mol. Biol. Evol. 28:2731-2739.

Truman, W., Sreekanta, S., Lu, Y., Bethke, G., Tsuda, K., Katagiri, F., and Glazebrook, J. 2013. The CALMODULIN-BINDING PROTEIN60 family includes both negative and positive regulators of plant immunity. Plant Physiol. 163:1741-1751. 
Varkonyi-Gasic, E., Wu, R., Wood, M., Walton, E. F., and Hellens, R. P. 2007. Protocol: A highly sensitive RT-PCR method for detection and quantification of microRNAs. Plant Methods 3:12.

Vlot, A. C., Dempsey, D. A., and Klessig, D. F. 2009. Salicylic acid, a multifaceted hormone to combat disease. Annu. Rev. Phytopathol. 47:177-206.

Wang, C., He, X., Wang, X., Zhang, S., and Guo, X. 2017a. ghr-miR5272amediated regulation of $G h M K K 6$ gene transcription contributes to the immune response in cotton. J. Exp. Bot. 68:5895-5906.

Wang, L., Tsuda, K., Sato, M., Cohen, J. D., Katagiri, F., and Glazebrook, J. 2009. Arabidopsis CaM binding protein CBP60g contributes to MAMPinduced SA accumulation and is involved in disease resistance against Pseudomonas syringae. PLoS Pathog. 5:e1000301.

Wang, L., Tsuda, K., Truman, W., Sato, M., Nguyen, V., Katagiri, F., and Glazebrook, J. 2011. CBP60g and SARD1 play partially redundant critical roles in salicylic acid signaling. Plant J. 67:1029-1041.

Wang, L., Wu, S. M., Zhu, Y., Fan, Q., Zhang, Z. N., Hu, G., Peng, Q. Z., and $\mathrm{Wu}$, J. H. 2017b. Functional characterization of a novel jasmonate ZIM-domain interactor (NINJA) from upland cotton (Gossypium hirsutum). Plant Physiol. Biochem. 112:152-160.

Wang, Q., Liu, N., Yang, X., Tu, L., and Zhang, X. 2016. Small RNAmediated responses to low- and high-temperature stresses in cotton. Sci. Rep. 6:35558.

Wang, Y. Q., Chen, D. J., Wang, D. M., Huang, Q. S., Yao, Z. P., and Liu, F. J. 2004. Over-expression of Gastrodia anti-fungal protein enhances Verticillium wilt resistance in coloured cotton. Plant Breed. 123: 454-459.
Xie, F., Wang, Q., Sun, R., and Zhang, B. 2015. Deep sequencing reveals important roles of microRNAs in response to drought and salinity stress in cotton. J. Exp. Bot. 66:789-804.

Yan, J., Gu, Y., Jia, X., Kang, W., Pan, S., Tang, X., Chen, X., and Tang, G. 2012. Effective small RNA destruction by the expression of a short tandem target mimic in Arabidopsis. Plant Cell 24:415-427.

Yin, Z., Li, Y., Han, X., and Shen, F. 2012. Genome-wide profiling of miRNAs and other small non-coding RNAs in the Verticillium dahliaeinoculated cotton roots. PLoS One 7:e35765.

Yuan, P., Jauregui, E., Du, L., Tanaka, K., and Poovaiah, B. W. 2017. Calcium signatures and signaling events orchestrate plant-microbe interactions. Curr. Opin. Plant Biol. 38:173-183.

Zhang, T., Zhao, Y. L., Zhao, J. H., Wang, S., Jin, Y., Chen, Z. Q., Fang, Y. Y., Hua, C. L., Ding, S. W., and Guo, H. S. 2016. Cotton plants export microRNAs to inhibit virulence gene expression in a fungal pathogen. Nat. Plants 2:16153.

Zhang, Y., Wang, W., Chen, J., Liu, J., Xia, M., and Shen, F. 2015a. Identification of miRNAs and their targets in cotton inoculated with Verticillium dahliae by high-throughput sequencing and degradome analysis. Int. J. Mol. Sci. 16:14749-14768.

Zhang, Z., Jiang, L., Wang, J., Gu, P., and Chen, M. 2015b. MTide: An integrated tool for the identification of miRNA-target interaction in plants. Bioinformatics 31:290-291.

Zhu, Q. H., Fan, L., Liu, Y., Xu, H., Llewellyn, D., and Wilson, I. 2013. miR482 regulation of $N B S-L R R$ defense genes during fungal pathogen infection in cotton. PLoS One 8:e84390. 